
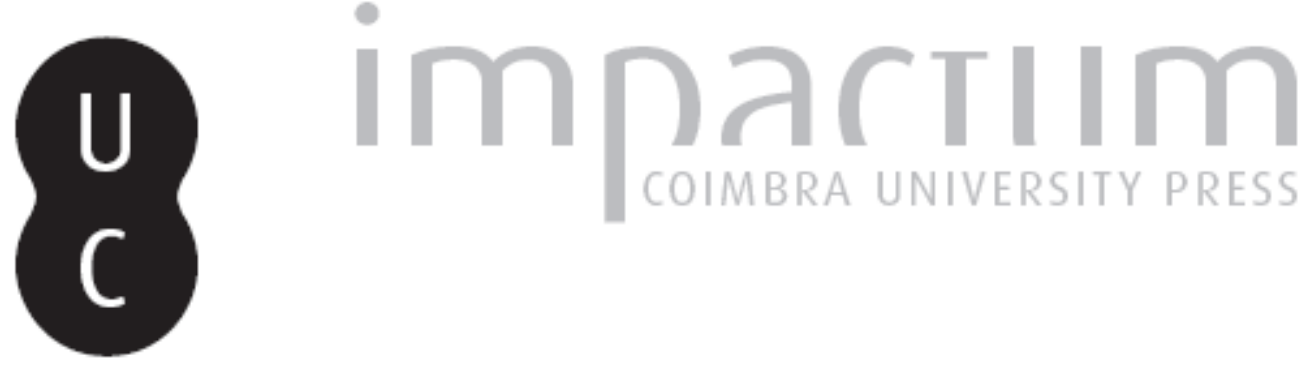

\title{
Sumários do Boletim Bibliográfico da Biblioteca da Universidade de Coimbra, Vol. I (1914) - vol. III (1916) e do Boletim da Biblioteca da Universidade de Coimbra, Vol. IV (1917) - vol. XLV (2014)
}

Publicado por: Imprensa da Universidade de Coimbra

URL

persistente: URI:http://hdl.handle.net/10316.2/43035

DOI: DOl:https://doi.org/10.14195/1647-8436_46_47_13

Accessed : $\quad$ 26-Apr-2023 16:19:51

A navegação consulta e descarregamento dos títulos inseridos nas Bibliotecas Digitais UC Digitalis, UC Pombalina e UC Impactum, pressupõem a aceitação plena e sem reservas dos Termos e Condições de Uso destas Bibliotecas Digitais, disponíveis em https://digitalis.uc.pt/pt-pt/termos.

Conforme exposto nos referidos Termos e Condições de Uso, o descarregamento de títulos de acesso restrito requer uma licença válida de autorização devendo o utilizador aceder ao(s) documento(s) a partir de um endereço de IP da instituição detentora da supramencionada licença.

Ao utilizador é apenas permitido o descarregamento para uso pessoal, pelo que o emprego do(s) título(s) descarregado(s) para outro fim, designadamente comercial, carece de autorização do respetivo autor ou editor da obra.

Na medida em que todas as obras da UC Digitalis se encontram protegidas pelo Código do Direito de Autor e Direitos Conexos e demais legislação aplicável, toda a cópia, parcial ou total, deste documento, nos casos em que é legalmente admitida, deverá conter ou fazer-se acompanhar por este aviso. 


\section{BOLETIM DA \\ BIBLIOTECA GERAL DA UNIVERSIDADE DE COIMBRA}

VOL. 46/47 (2015/2016)

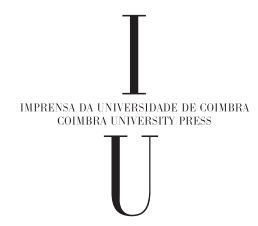




\section{Sumários do Boletim Bibliográfico da Biblioteca da Universidade de} Coimbra, Vol. I (1914) - vol. III (1916) e do Boletim da Biblioteca da

\section{Universidade de Coimbra, Vol. IV (1917) - vol. XLV (2014)}

\section{Vol. I (1914)}

SOUSA, Marnoco e - Preâmbulo, p. 1-3

RELAÇÃO DAS PUBLICAÇÕES portuguesas recebidas nesta Biblioteca durante o mês de dezembro de 1913, p. 4-23

CATÁLOGO DOS MANUSCRITOS da Biblioteca da Universidade de Coimbra, p. 24-27, 74-77, 136-139, 181-183, 227-229, 285-287, 324-325, 370-375, 422-425, 468-469, 514-518, 562-565

CASTRO, A. M. Simões de - Um manuscrito interessante de D. Marcos da Cruz, p. 28-35

SOUSA, Marnoco e - Censo da população de 1911, p. 36-39

CARVALHO, Teixeira de - Livraria do Mosteiro de Santa Cruz de Coimbra, p. 40-52, 96-110, 148-158, 195-206, 242-254, 295-302, 384-388, 575-582 RELAÇÃO DAS PUBLICAÇÕES portuguesas recebidas nesta Biblioteca durante o mês de janeiro de 1914, p. 53-73

CASTRO, A. M. Simões de - Vinda de El-rei D. João 3. ${ }^{\circ}$ a Coimbra no ano de 1550, p. 78-86, 140-147, 191-194, 291-294

SILVA, Luciano Pereira da - O «Libro de álgebra» de Pedro Nunes, p. 87-95 RELAÇÃO DAS PUBLICAÇÕES portuguesas recebidas nesta Biblioteca durante o mês de fevereiro de 1914, p. 111-135 
RELAÇÃO DAS PUBLICAÇÕES portuguesas recebidas nesta Biblioteca durante o mês de março de 1914, p. 159-180

SILVA, Henriques da - Dissertação de licenciatura de Direito, p. 184-190, p. 230-235, 288-290, 326-328, 378-383, 426-430, 470-472, 519-526, 566-568 RELAÇÃO DAS PUBLICAÇÕES portuguesas recebidas nesta Biblioteca durante o mês de abril de 1914, p. 207-226

CASTRO, A. M. Simões de - Frontispício ornamentado de um exemplar manuscrito em pergaminho da «Chronica de D. Afonso Henriques» de Duarte Galvão, p. 236-241

RELAÇÃO DAS PUBLICAÇÕES portuguesas recebidas nesta Biblioteca durante o mês de maio de 1914, p. 255-284

RELAÇÃO DAS PUBLICAÇÕES portuguesas recebidas nesta Biblioteca durante o mês de junho de 1914, p. 303-323

VASCONCELOS, Carolina Michaelis de - D. Francisco Manuel de Melo. Notas relativas a manuscritos da Biblioteca da Universidade de Coimbra, p. 329-346

CASTRO, A. M. Simões de - Alguns apontamentos acerca da 2. a edição dos «Diálogos de vária história» de Pedro de Mariz, p. 347-350

RELAÇÃO DAS PUBLICAÇÕES portuguesas recebidas nesta Biblioteca durante o mês de julho de 1914, p. 351-369

CARVALHO, Teixeira de - Pedro de Mariz e a Livraria da Universidade de Coimbra, p. 389-398, 438-446, 482-494, 533-542

RELAÇÃO DAS PUBLICAÇÕES portuguesas recebidas nesta Biblioteca durante o mês de agosto de 1914, p. 399-421

SOUSA, Marnoco e - Estatística da Biblioteca da Universidade no ano lectivo de 1913-1914, p. 431-437

RELAÇÃO DAS PUBLICAÇÕES portuguesas recebidas nesta Biblioteca durante o mês de setembro de 1914, p. 447-467

CASTRO, A. M. Simões de - A «Vita Christi» da Biblioteca da Universidade de Coimbra, p. 473-481

RELAÇÃO DAS PUBLICAÇÕES portuguesas recebidas nesta Biblioteca durante o mês de outubro de 1914, p. 495-513

SILVA, Luciano Pereira da - O «Regimento do estrolábio» da Biblioteca Real de Munich, p. 527-532

RELAÇÃO DAS PUBLICAÇÕES portuguesas recebidas nesta Biblioteca durante o mês de novembro de 1914, p. 543-561 
A GENTE DE NAÇÃO e a Inquisição de Portugal no último quartel do século

17, p. $569-574$

ÍNDICE do $1^{\circ}$ ano, p. 583-584

ÍNDICE das Gravuras, p. 584-585

\section{Vol. II (1915)}

SOUSA, Marnoco e - Preâmbulo, p. I

CATÁLOGO DOS MANUSCRITOS da Biblioteca da Universidade de Coimbra (continuação do vol. I), p. 1-4, 33-36, 65-68, 89-92, 117-120, 145-148, 169172, 233-235, 265-268, 293-295, 325-328

SILVA, Dias da - Das prescrições de curto prazo. Dissertação de Licenciatura em Direito, p. 5-8, 37-47, 69-70, 93-95, 121-124, 149-151, 173-175, 197-202, 236-241, 269-271, 296-298

MATA, Caeiro da - Os estudos de história do Direito e a obra do Sr. Dr. Gama Barros, p. 9-14

CARVALHO, Teixeira de - Livraria do Mosteiro de Santa Cruz de Coimbra (continuação do vol. I), p. 15-19, 48-52, 71-80, 283-292, 321-324, 350-358

VASCONCELOS, Carolina Michaelis de - D. Francisco Manuel de Melo. Notas relativas a manuscritos da Biblioteca da Universidade de Coimbra, $\mathrm{p}$. 20-32, 53-64

MATA, Caeiro da - Os forais de Almada, p. 81-88, 96-98, 125-126, 152-154, 176-178, 203-205, 242-245, 272-273

CASTRO, A. M. Simões de - As constituições do bispado de Coimbra publicadas em 1521 pelo bispo conde D. Jorge de Almeida e notas biográficas a ele relativas, p. 99-109, 127-134, 155-162, 179-187, 206-215, 246-256, 274-282, 303-310, 337-344

CARVALHO, Teixeira de - Um livro raro, p. 110-116, 135-144, 163-168, 188196, 216-221, 257-264

SILVA, Luciano Pereira da - $\mathrm{O}$ «Tratado del esphera y del arte del marear» de Francisco Faleiro, p. 222-232

CARVALHO, Teixeira de - Livraria do Mosteiro de Santa Cruz de Coimbra (continuação do vol. I), p. 283-292, 321-324, 350-358

CARVALHO, Teixeira de - Um manuscrito de João Pedro Ribeiro «Extractos para servirem a ordenar-se o Glozario Latino-Lusitano e Archeologico Portuguez, contendo tãobem algũas notícias históricas», p. 299-302, 329-336 
CARVALHO, Teixeira de - Notas de um escrivão do povo, p. 311-320, 345-349

ÍNDICE do $2^{\circ}$ ano, p. 359-360

ÍNDICE de gravuras, p. 360

ERROS e aditamentos, p. 361

RELAÇÃO DAS PUBLICAÇÕES portuguesas recebidas nesta Biblioteca no mês de dezembro de 1914 a dezembro de 1915, p. I-CXLVII

\section{Vol. III (1916)}

CATÁLOGO DOS MANUSCRITOS da Biblioteca da Universidade de Coimbra (cont. do vol. II), p. 1-3, 45-49, 114-118, 234-240

SILVA, Dias da - Das prescrições de curto prazo. Dissertação de Licenciatura em Direito (cont. do vol. II), p. 4-9, 50-57, 119-126, 241-247

CARVALHO, Teixeira de - Um manuscrito de João Pedro Ribeiro «Extractos para servirem a ordenar-se o Glozario Latino-Lusitano e Archeologico Portuguez, contendo tãobem algũas notícias históricas» (cont. do vol. II), p. 10-19, 151-164, 290-298

CASTRO, A. M. Simões de - As constituições do bispado de Coimbra publicadas em 1521 pelo bispo conde D. Jorge de Almeida e notas biográficas a ele relativas (cont. do vol. II), p. 20-27, 58-72, 127-133, 248-253

GUIMARÃES, Rodolfo - A edição de 1546 do Livro de Pedro Nunes «De arte atque ratione navigandi», p. 28-36

SOUSA, Marnoco e - Movimento da Biblioteca da Universidade de Coimbra no ano de 1915, p. 37

SANTOS, Alves dos - Advertência, p. 39-40

SANTOS, Alves dos - Doutor Marnoco e Sousa, p. 41-44

CARVALHO, Teixeira de - Livraria do Mosteiro de Santa Cruz de Coimbra (cont. do vol. II), p. 73-82

PARA A HISTÓRIA do ensino público em Portugal (um documento importante), p. 83-113

CARVALHO, Teixeira de - Notas de um escrivão do povo (cont. do vol. II), p. 134-140, 254-267

SANTOS, Alves dos - Estatística numérica e gráfica do movimento da Biblioteca da Universidade de Coimbra no $1^{\circ}$ semestre de 1916, p. 141-150 SANTOS, Alves dos - Novo Catálogo Metódico da Biblioteca da Universidade de Coimbra, 165-174 
SANTOS, Alves dos - O «crescimento» da criança portuguesa (Subsídios para a constituição duma Pedologia nacional), p. 175-233

GUIMARÃES, Rodolfo - Um opúsculo raríssimo de Pedro Nunes, p. 268-289 SANTOS, Alves dos - Estatística do movimento da Biblioteca da Universidade de Coimbra no $2^{\circ}$ semestre de 1916, p. 299-300

RELAÇÃO DAS PUBLICAÇÕES recebidas na Biblioteca no mês de dezembro de 1915 a novembro de 1916, p. I-LXXIII

ÍNDICE do $3^{\circ}$ ano, p. LXXV-LXXVI

ÍNDICE das Gravuras, p. LXXVI

\section{Vol. IV (1917)}

SANTOS, Alves dos - O «crescimento» da criança portuguesa (Subsídios para a constituição duma Pedologia nacional) (cont. do vol. III), p. 1-59

CATÁLOGO DOS MANUSCRITOS da Biblioteca da Universidade de Coimbra (cont. do vol. III), p. 61-65, 143-149

CASTRO, A. M. Simões de - As constituições do bispado de Coimbra publicadas em 1521 pelo bispo conde D. Jorge de Almeida e notas biográficas a ele relativas (cont. do vol. III), p. 66-75, 150-158

CARVALHO, Teixeira de - Um manuscrito de João Pedro Ribeiro «Extractos para servirem a ordenar-se o Glozario Latino-Lusitano e Archeologico Portuguez, contendo tãobem algũas notícias históricas» (cont. do vol. III), p. 76-84, 169-174

GUIMARÃES, Rodolfo - O livro de Vernier, p. 85-97, 224-255

VERNIER, Pedro - La constrvction, I'vsage, et les proprietes dv qvadrant novveav de mathematique, p. 98-121

SILVA, Luciano Pereira da - «O Diálogo em louvor da nossa linguagem» de João de Barros, p. 122-139

SANTOS, Alves dos - Estatística do movimento da Biblioteca da Universidade de Coimbra no $1^{\circ}$ semestre de 1917, p. 140-142

CASTRO, Augusto Mendes Simões de - Alguns escritos do cardeal D. Jorge da Costa, p. 159-168

DONATO, Ernesto - Os desacatos em Portugal: o desacato da rial capela, no tempo de D. João III (À margem duma Provisão), p. 175-194

CARVALHO, Teixeira de - Notas de um escrivão do povo (cont. do vol. III), p. $195-211$ 
DONATO, Ernesto - Dissertações da Universidade de Coimbra, p. 212-223

SANTOS, Alves dos - Estatística do movimento da Biblioteca da Universidade de Coimbra no $2^{\circ}$ semestre de 1917, p. 256-258

RELAÇÃO DAS PUBLICAÇÕES recebidas na Biblioteca nos meses de dezembro de 1916 a dezembro de 1917, p. I-LXIV

ÍNDICE do $4^{\circ}$ ano, p. LXV-LXVI

ÍNDICE de Gravuras, p. LXVI

\section{Vol. V (1918) publicado em 1920}

CASTRO, Augusto Mendes Simões de - Catálogo dos Manuscritos da Biblioteca da Universidade de Coimbra (cont. do vol. IV), p. 1-11

CASTRO, A. M. Simões de - As constituições do bispado de Coimbra publicadas em 1521 pelo bispo conde D. Jorge de Almeida e notas biográficas a ele relativas (cont. do vol. IV), p. 12-23

ALGUNS ESCRITOS do cardeal D. Jorge da Costa (continuação do vol. IV), p. 24-47

DONATO, Ernesto - Manuscritos de João Pedro Ribeiro, p. 48-60

FERREIRA, A. Aurélio da Costa - A arte de educar e a psicologia experimental, p. 61-69

CARVALHO, Teixeira de - Um manuscrito de João Pedro Ribeiro «Extractos para servirem a ordenar-se o Glozario Latino-Lusitano e Archeologico Portuguez, contendo tãobem algũas notícias históricas» (cont. do vol. IV), p. 70-77

OLIVEIRA, J. I. de - Um equívoco bibliográfico, p. 78-81

DONATO, Ernesto - Catálogo das Dissertações da Universidade de Coimbra (cont. do vol. IV), p. 82-95

CARVALHO, Teixeira de - Compromisso da Misericórdia de Coimbra, p. 96-153

REGIMENTO DAS OBRAS da Universidade, p. 154-174

CARVALHO, Teixeira de - Notas de Camilo Castelo Branco num livro que Ihe pertenceu, p. 175-219

SANTOS, Alves dos - Mapa do movimento da Biblioteca Geral da Universidade de Coimbra no ano de 1918, p. 221

RELAÇÃO DAS PUBLICAÇÕES recebidas na Biblioteca nos meses de janeiro a dezembro de 1918, p. I-LXXIV 
ÍNDICE do $5^{\circ}$ ano, p. LXXV-LXXVI

ÍNDICE das Gravuras, p. LXXVI

CORRIGENDA, p. LXXVI

\section{Vol. VI (1919-1921)}

SANTOS, Alves dos - Assistência às crianças normais, de ambos os sexos, desde os 7 aos 18 anos, em perigo moral, p. 1-17

PROJECTO DE LEl sobre a reorganização do Ensino Público, p. 18-42

CASTRO, Augusto Mendes Simões de - Catálogo dos Manuscritos da Biblioteca da Universidade de Coimbra (cont. do vol. V), p. 43-49

DONATO, Ernesto - Manuscritos de João Pedro Ribeiro (continuação do vol. V), p. 50-64

SILVA, Luciano Pereira da - O «Regimento do Estrolábio» da Biblioteca de Évora (cont. do vol. I), p. 65-79

UM EQUÍVOCO bibliográfico [«Relaçam feita em Consistorio secreto... sobre a vida, \& Sanctidade, actos de Canonizaçam, \& milagres da Beata Isabel Raynha de Portugal...»], p. 80-117

SANTOS, Alves dos - Alocução proferida pelo Presidente da Câmara Municipal de Coimbra...na comemoração do quarto centenário de Fernando de Magalhães, p. 118-120

HENRIQUES, Júlio A. - Dr. Joaquim Martins Teixeira de Carvalho, p. 121-122

CARVALHO, Teixeira de - «Comédia Eufrosina», de Jorge Ferreira de Vasconcelos. Notas à margem do recente estudo do sr. Aubrey F. G. Bell, sobre a edição de 1561, p. 123-157

DONATO, Ernesto - Catálogo das Dissertações da Universidade de Coimbra (cont. do vol. V), p. 158-171

SANTOS, Alves dos - Discurso de recepção dos Marechais Joffre, Diaz e Smith Dorrien, na Câmara Municipal de Coimbra, em 15 de Abril de 1921, p. 172-174

SANTOS, Alves dos - Discurso de recepção dos delegados estrangeiros à Conferência Interparlamentar de Comércio, na Câmara Municipal de Coimbra, em 27 de Abril de 1921, p. 175-177

SANTOS, Alves dos - Subsídio de 50 contos concedido à Biblioteca, p. 178-182 UM MANUSCRITO de Manuel de Faria Severim?, p.183-189 
CARVALHO, Joaquim de - A erudição de Gomes Eannes de Zurara (Notas em torno de alguns plágios deste Cronista), p. 190-201

MEMORIAS DOS ESTUDOS, em que se criarão os monges de S. Jeronymo, e suas mudanças desde o tempo da sua fundação em Portugal, athe o feliz reynado do Fidelissimo Sñr. Rey D. José o primeyro que Deos Guarde, p. 202-276

CASTRO, Augusto Mendes Simões de - Notas acerca da vinda e estada de El-Rei Dom Sebastião em Coimbra no ano de 1570 e do modo como foi recebido pela Universidade, p. 277-322

REGULAMENTO DA BIBLIOTECA, p. 323-331

SANTOS, Alves dos - Mapa do movimento da Biblioteca Geral da Universidade de Coimbra no ano de 1919, p. 333

SANTOS, Alves dos - Mapa do movimento da Biblioteca Geral da Universidade de Coimbra no ano de 1920, p. 335

SANTOS, Alves dos - Mapa do movimento da Biblioteca Geral da Universidade de Coimbra no ano de 1921, p. 337

ÍNDICE do vol. VI (1919-1921), p. 339-340

ÍNDICE das Gravuras, p. 341

\section{Vol. VII (1922) publicado em 1925}

SANTOS, Alves dos - A medida em Psicologia, p. 1-25

SANTOS, Alves dos - Laboratório de Psicologia Experimental, p. 26-41

SANTOS, Alves dos - Catálogo dos Cimélios da nossa Biblioteca: breve conversação preambular, p. 42-48

DONATO, Ernesto - Plano deste Catálogo [dos Cimélios], p. 50-68

CASTRO, Augusto Mendes Simões de - Catálogo dos Manuscritos (continuação do vol. VI), p. 69-80

DONATO, Ernesto - Manuscritos de João Pedro Ribeiro (continuação do vol. VI), p. 81-99

SILVA, Luciano Pereira da - O «Regimiento de navegacion» de Pedro de Medina, Sevilla, 1552, p. 100-113

CARVALHO, Joaquim de - A erudição de Gomes Eannes de Zurara (notas em torno de alguns plágios deste cronista) (cont. do vol. VI), p. 114-140 TRANCOSO, Gonçalo Fernandes - «Regra geral pera aprender a tirar pola mão as festas mudaueis...», p. 141-182 
SILVA, Luciano Pereira da - A «Regra geral das festas mudáveis» de Gonçalo Trancoso, autor dos «Contos de proveito e exemplo», p. 183-210 PIMENTA, Belisário - O padroado da igreja de Miranda do Corvo nos manuscritos da Biblioteca da Universidade (1735-1737), p. 211-232

MEMORIAS DOS ESTUDOS, em que se criarão os monges de S. Jeronymo, e suas mudanças desde o tempo da sua fundação em Portugal, athe o feliz reynado do Fidelissimo Sñr. Rey D. José o primeyro que Deos Guarde (continuação), p. 233-257

UM MANUSCRITO de João Pedro Ribeiro «Extractos para servirem a ordenar-se o Glozario Latino-Lusitano e Archeologico Portuguez...» (cont. do vol. V), p. 258-263

DONATO, Ernesto - Vasco da Gama na Biblioteca Geral da Universidade: uma exposição bibliográfica, p. 264-285

DONATO, Ernesto - Movimento da Biblioteca Geral da Universidade de Coimbra: anos de 1922 a 1925, p. 286

ÍNDICE do vol. VII (1922-1925), p. 287-288

ÍNDICE das Gravuras, p. 288

\section{Vol. VIII (1926-1927) publicado em 1928}

CARVALHO, Joaquim de - A Livraria dum letrado do século XVI: Frei Diogo de Murça, p. 1-26

CASTRO, Augusto Mendes Simões de - A Universidade de Coimbra e o Marquês de Pombal, p. 27-33

COELHO, P. M. Laranjo - A Biblioteca Municipal de Castelo de Vide (História de uma Livraria), p. 34-57

DONATO, Ernesto - Catálogo dos cimélios da nossa Biblioteca (continuação do vol. VII), p. 58-69, 368-378

DONATO, Ernesto - Um manuscrito de João Pedro Ribeiro «Extractos para servirem a ordenar-se o Glozario Latino-Lusitano e Archeologico Portuguez...» (cont. do vol. VII), p. 70-75, 323-335

MADAHIL, A. G. da Rocha - Os Sonetos à morte de Francisco Rodrigues Lobo, p. 76-83

DONATO, Ernesto - Catálogo das Dissertações da Universidade de Coimbra (cont. do vol. VI), p. 84-88, 336-357 
MADAHIL, A. G. da Rocha - O Catálogo dos Manuscritos da Biblioteca da Universidade (cont. do vol. VII), p. 89-130, 299-322

DONATO, Ernesto - Manuscritos de João Pedro Ribeiro (cont. do vol. VII),

p. 131-137, 360-367

NEVES, Álvaro - Memórias biográficas de Joaquim Heliodoro da Cunha Rivara, p. 138-154, 215-298

RELAÇÃO DAS PUBLICAÇÕES portuguesas e estrangeiras recebidas nesta Biblioteca, de janeiro a junho de 1927, p. 155-195

RELAÇÃO DAS PUBLICAÇÕES portuguesas e estrangeiras recebidas nesta Biblioteca, de julho a dezembro de 1927, p. I-CXIII

MACEDO, Gomes de - Remonstrance faicte aux Etats Generaux des Provinces unies du Pays-Bas par l'Ambassadeur du Roy de Portugal, le XIX d'octobre 1587, p. 197-213

GUERRA, L. de Figueiredo da - Problema bibliográfico, p. 358-359

MADAHIL, A. G. da Rocha - Os códices de Santa Cruz de Coimbra, p. 379-420 DONATO, Ernesto - A Livraria de João Pedro Ribeiro legada à Universidade de Coimbra em 1839, p. 421-438

CARVALHO, Joaquim de - Catálogo dos professores de filosofia do Colégio das Artes de Coimbra e da Universidade de Évora desde 1555 a 1667, p. 439-448

MELO, J. B. Pereira de - Notas subsidiárias para uma bibliografia portuguesa da Grande Guerra, p. 449-469

CARVALHO, Joaquim de - Movimento da Biblioteca Geral da Universidade de Coimbra: anos de 1926 e 1927, p. 470-471

NOTICIÁRIO, p. 472

ÍNDICE do vol. VIII (1927), p. 473-474

ÍNDICE das Gravuras, p. 474

ÍNDICE por Autores, p. 474-475

\section{Vol. IX (1928) publicado em 1930}

RELAÇÃO DAS PUBLICAÇÕES portuguesas e estrangeiras recebidas nesta Biblioteca, de janeiro a junho de 1928, p. I-CXL

JEANROY, A. - Un «Cancionero» inconnu à la Bibliothèque de I'Université de Coimbra, p. 1-5 
DIAS, João Pereira - Biblioteca Matemática (Anexa à Faculdade de Sciências de Coimbra): Relatório..., p. 6-11

NEVES, Álvaro - Memórias biográficas de Joaquim Heliodoro da Cunha Rivara (cont. do vol. VIII), p. 12-87, 281-342

BARBOSA, António - Dois inéditos de João Baptista Lavanha, p. 88-93

DONATO, Ernesto - Os Vilhancicos da Biblioteca Geral da Universidade de Coimbra, p. 94-144, 384-497

MADAHIL, A. G. da Rocha - Catálogo dos Manuscritos da Biblioteca da Universidade, p. 145-180; 440-465

DONATO, Ernesto - Um manuscrito de João Pedro Ribeiro (cont. do vol. VIII), p. 181-191, 498-505

MADAHIL, A. G. da Rocha - Os códices de Santa Cruz de Coimbra (cont. do vol VIII), p. 192-229, 352-383

DONATO, Ernesto - Catálogo dos cimélios da nossa Biblioteca (cont. do vol. VIII), p. 230-240

MELO, José Brandão Pereira de - Notas subsidiárias para uma bibliografia portuguesa da Grande Guerra (cont. do vol VIII), p. 241-272; 466-497

DONATO, Ernesto - Catálogo das Dissertações da Universidade de Coimbra (cont. do vol. VIII), p. 273-280

DONATO, Ernesto - Manuscritos de João Pedro Ribeiro, p. 343-351

ÍNDICE do vol. IX (1928), p. 507

ÍNDICE de Autores, p. 508

ÍNDICE das Gravuras, p. 508

RELAÇÃO DAS PUBLICAÇÕES portuguesas e estrangeiras recebidas nesta Biblioteca, de julho de 1928 a junho de 1929, p. I-CCLXIII

\section{Vol. X (1932) publicado em 1933}

RELAÇÃO DAS PUBLICAÇÕES portuguesas e estrangeiras recebidas nesta Biblioteca, de janeiro a junho de 1929, p. I-CCIII

LIMA, Henrique de Campos Ferreira de - Exposição bibliográfica e iconográfica comemorativa do $1 .^{\circ}$ centenário da batalha da Vila da Praia, em 11 de Agosto de 1829, p. 1-54

MADAHIL, A. G. da Rocha - Os códices de Santa Cruz de Coimbra (cont. do vol. IX), p. 55-105 
MELO, José Brandão Pereira de - Notas subsidiárias para uma bibliografia portuguesa da Grande Guerra (cont. do vol. IX), p. 106-154

DONATO, Ernesto - Catálogo dos cimélios da nossa Biblioteca (cont. do vol. IX), p. 155-160

MADAHIL, A. G. da Rocha - A Biblioteca da Universidade de Coimbra e as suas marcas bibliográficas, p. 161-231

DONATO, Ernesto - Um manuscrito de João Pedro Ribeiro (cont. do vol. IX), p. 232-239

COSTA, Júlio Dias da - Uma tradução falsamente atribuída a Camilo, p. 240-249

MADAHIL, A. G. da Rocha - Catálogo dos Manuscritos da Biblioteca da Universidade de Coimbra (cont. do vol. IX), p. 250-308

REGULAMENTO da Biblioteca matemática anexa à 1 a Secção da Faculdade de Ciências da Universidade de Coimbra, p. 309-324

CASTRO, Augusto Mendes Simões de - Um folheto muito raro, p. 325-326 «TESTAMENTO e ultima disposiçam com que faleceo o lecenceado Manoel

Soares d'Oliveira...», p. 327-342

MOVIMENTO DA BIBLIOTECA Geral da Universidade de Coimbra: anos de 1928 a 1932 , p. 343-348

ÍNDICE do vol. X (1932), p. 349

ÍNDICE por Autores, p. 350

ÍNDICE das Gravuras, p. 350

\section{Vol. XI (1933) publicado em 1934}

COSTA, João da Providência - Dr. J. Mendes dos Remédios (1867-1932), p. V-IX DONATO, Ernesto - Os «Reservados» da Biblioteca da Universidade de Coimbra, p. 1- 49

MADAHIL, A. G. da Rocha - Os códices de Santa Cruz de Coimbra (cont. do vol. X), p. 50-96

DONATO, Ernesto - Manuscritos de João Pedro Ribeiro (cont. do vol. X), p. 97-110; pp. 254-262

CRUZ, António - Catálogo dos Manuscritos da Biblioteca da Universidade de Coimbra (cont. do vol. X), p. 111-194

DONATO, Ernesto - Catálogo dos cimélios da nossa Biblioteca (cont. do vol. X), p. 195-202 
CAMPOS, Agostinho de - «Menina e moça» de Bernardim Ribeiro: confronto das edições de 1554 e 1559 por Joaquim Freire. Nota Prévia, p. 203-208 FREIRE, Joaquim - Bernardim Ribeiro, «Menina e moça» : Cotejo da ed. de 1554 com a de 1559 , p. 209-222

DONATO, Ernesto - Catálogo das Dissertações da Universidade de Coimbra (cont. do vol. IX), p. 223-227

MELO, José Brandão Pereira de - Notas subsidiárias para uma bibliografia portuguesa da Grande Guerra (cont. do vol. X), p. 228-253

DONATO, Ernesto - Um manuscrito de João Pedro Ribeiro (cont. do vol. IX), p. 254-262

DONATO, Ernesto - Ex-Libris nacionais e estrangeiros. A «Colecção J. Jardim de Vilhena», p. 263-279

MOVIMENTO DA BIBLIOTECA Geral da Universidade de Coimbra no ano de 1933, p. 280-281

ÍNDICE do vol. XI (1933), p. 283

ÍNDICE por Autores, p. 284

ÍNDICE das Gravuras, p. 284

Vol. XI (1934) - Suplementos

CATÁLOGO DAS REVISTAS nacionais e estrangeiras recebidas nas principais bibliotecas de Coimbra, p. 5-124

CATÁLOGO DOS LIVROS de medicina dos sécs. XV e XVI expostos por ocasião do III Congresso Internacional de História das Ciências, p. 1-64

\section{Vol. XII (1934-1935) publicado em 1936}

BRITO, A. da Rocha - «Tratado sobre a provincia D'Antre Douro e Minho e suas avondanças, copilado por Mestre Antonio fisiquo e çolorgiam morador na vila de Guimarães e natural della», p. 1-10

MADAHIL, A. G. da Rocha - «A Visitaçam geral do estado espiritual desta See de Coibra, de 1556», p. 11-113

CRUZ, António - Um inédito de António Coelho Gasco sobre antiguidades de Trás-os-Montes, p. 114-119

ANTIQUÁRIO DISCURSO dedicado ao Ill.mo e R.mo S.or D. Rodrigo da Cunha, Arcebp..$^{\circ}$ de Braga, S.or della, primas das Hespanhas, e elleito metropolitano de Lisboa, p. 120-137 
DESCRIPÇÃO BURLESCA de um imaginario aeróstato e de seus petrechos, satyra ao P.e Bartholomeu Lourenço de Gusmão, p. 138-148

FERNÁNDEZ ALMUZARA, Eugenio - El Manuscrito 726 de la Biblioteca de la Universidad de Coimbra, o la "Historia de varios Reyes de Castilla», p. 149-165

DONATO, Ernesto - Os «Reservados» da Biblioteca da Universidade de Coimbra (cont. do vol. XI), p. 166-177

O CASAMENTO DE D. PEDRO II com a Princesa de Neuburg (documentos diplomáticos), p. 178-207

MOVIMENTO da Biblioteca Geral da Universidade de Coimbra: ano de 1934, p. 208

ÍNDICE ao vol. XII (1934-1935), p. 209

ÍNDICE de autores, p. 209-210

ÍNDICE de gravuras, p. 210

\section{Vol. XII (1935) - Suplemento - vol. I}

COSTA, J. da Providencia - Duas palavras de apresentação, p. V-VII

BEAU, Albin Eduard - Antero de Quental e a ideia da morte, p. 1-23

BRITO, A. da Rocha - O primeiro dia d'aula, a primeira casa da Faculdade, p. 24-27

- O primeiro lente, o primeiro livro de medicina, p. 28-43

- Os primeiros alunos, p. 44-76

- As primeiras sebentas, p. 77-78

- O primeiro bacharel em medicina, p. 79-80

- O primeiro licenciado na Faculdade, p. 81-93

- O primeiro concurso para professor na Faculdade de Medicina, p. 94-103

- O primeiro doutor, p. 104-109

- O primeiro boticário, p. 110

- O primeiro sangrador, p. 111-115

- O primeiro bedel, p. 116-121

NOTAS E DOCUMENTOS:

Quadro do pessoal universitário, nos primeiros anos, p. 123-125

Dom Garcia d'Almeida, nosso primeiro Reitor, p. 126

Os cancelários, p. 127-132

Primeira eleição de conselheiros, p. 133 
Primeira eleição de deputados, p. 133

O primeiro conservador, p. 134-135

Primeira reunião a que assiste Henrique de Cuellar, p. 136

O primeiro recebedor, p. 137

O primeiro síndico, p. 138

Os primeiros taxadores, p. 139

Capelão da Universidade, p. 140

O primeiro meirinho, p. 140

O primeiro porteiro da Universidade, p. 140-143

Afinal a "Filantrópica" é muito velha, p. 144-146

Votos, p. 147

Agulhas, linhas e escrutínio secreto, p. 147

O primeiro lente de música, p. 148

Trombetas e charamelas, p. 149-152

O custo da vida em Quinhentos, p. 153-156

Briga de lentes, p. 157-158

Francisco do Amaral, culpado, p. 159

Acordo sobre balanças do carniceiro, almotacés e preços dos barretes e luvas, p. 160

A cidade e a Universidade, p. 160-162

Crise de habitação, p. 163-164

Amabilidades filipinas, p. 165

A Câmara e a Universidade, p. 166-169

As primeiras aposentações por velhice, invalidez e doença, p. 170-172

PINA, Luís de - A medicina portuguesa de além-mar no século XVI, p.

$173-204$

FLORES, Joaquina - Evolução do Senhor da Serra, p. 205-234

ÍNDICE, p. 237-238

\section{Vol. XII (1935) - Suplemento - vol. II}

PIMENTA, Alfredo - A evolução dum pensamento, p. 3-32

MOREIRA, José Carlos Martins - Política colonial, p. 33-49

SALGUEIRO, Trindade - O enigma humano, p. 50-76

BOLÉO, Manuel de Paiva - Língua falada, lógica e clássicos, p. 77-94

SANTOS, Maria dos - Thomson na literatura europeia, p. 95-137 
SOARES, Teixeira - Factores históricos da formação da unidade brasileira,

p. 138-156

NAVARRO, Saul de - Rapsódia brasileira, p. 157-218

MEIRELES, Cecília - Notícia da poesia brasileira, p. 219-268

ÍNDICE, p. 269

\section{Vol. XIII (1936) - Suplemento - vol. III}

NEMÉSIO, Vitorino - Relações francesas do romantismo português, p. 1-172 FERNÁNDEZ ALMUZARA, Eugênio - Relaciones de la épica de Lope de Vega y la de Camões, p. 173-192

QUEIRÓS, Francisco de - António Feijó e os poetas contemporâneos da Ribeira Lima, p. 193-259

ÍNDICE, p. 261

\section{Vol. XIII (1936) - Suplemento - vol. IV}

BOLÉO, Manuel de Paiva - O bucolismo de Teócrito e de Vergílio, p. 1-99 SOARES, Teixeira - Imagens de Machado de Assis, p. 100-127 CAMPOS, Agostinho de - Estudos sobre o soneto, p. 128-211 ALBUQUERQUE, Maria Manuela Barroso Henriques da Silva de - A ode na Grécia, p. 212-227

ÍNDICE, p. 229

\section{Vol. XIII (1936) - Suplemento - vol. V}

PEREIRA, M. Serras - Ensaios sobre a ideia de Deus, p. 3-32

TAMAGNINI, Eusébio - Alguns aspectos do problema escolar português, p. 33-119

FIGUEIREDO, Mário de - Princípios essenciais do Estado Novo Corporativo, p. $120-141$

PEREIRA, M. Serras - Deus como primeira fonte e último fundamento dos valores éticos, p. 142-178

FLASCHE, Han - Max Scheller: alguns aspectos da sua filosofia, p. 179-198 CHAIX-RUY, Jules - Les caractères dans I'oeuvre de François Mauriac, p. 199-231 ÍNDICE, p. 233 


\section{Vol. XIII (1936) - Suplemento - vol. VI}

WAGNER, Max L. - Restos de latinidad en el norte de África, p. 1-37

CAIRES, Álvaro Guimarães de - Esboço histórico da medicina dos portugueses no estrangeiro, p. 38-65

ROSS, E. Denison - António de Gouveia, p. 66-93

MACHADO, L. Saavedra - O pensamento inglês em Portugal na Idade Média, p. 94-123

BOLÉO, Manuel de Paiva - O perfeito e o pretérito em português em confronto com as outras línguas românicas, p. 124-266

ÍNDICE, p. 267

\section{Vol. XIII (1937)}

DIOGO DO COUTO. Década Quinta da «Ásia»: Texte inédit, publié d'après un manuscrit de la Bibliothèque de I'Université de Leyde, p. 1-752

\section{Vol. XIV (1938)}

BRANDÃO, Mário - Contribuições para a História da Universidade de Coimbra. Alguns documentos respeitantes à Universidade de Coimbra na época de D. João III, p. 1-232

DONATO, Ernesto - Os «Reservados» da Biblioteca da Universidade de Coimbra (continuação do vol. XII), p. 233-268

CRUZ, António - Subsídios para a História da Guerra da Aclamação, p. 269-305

VARIA, p. 306

ÍNDICE do vol. XIV, p. 309

ÍNDICE por Autores, p. 309

MOVIMENTO DA BIBLIOTECA Geral da Universidade de Coimbra: anos de $1935,1936,1937,1938$, p. 310-312

\section{Vol. XIV (1938) - Suplemento}

DONATO, Ernesto - No $1^{\circ}$ Centenário da morte do Mestre da Diplomática Portuguesa Doutor João Pedro Ribeiro, p. I-XIX 
CRUZ, António - Breve estudo dos manuscritos de João Pedro Ribeiro, p.

$1-239$

ÍNDICE geral, p. 240

\section{Vol. XV (1942)}

PERES, Damião - Palavras de abertura, p. V-VI

PERES, Damião - Notícia prévia, p. VII- VIII

Vida «e acçoes» do grande D. Ant. ${ }^{\circ}$ Luis de M.ez, terceyro Conde de Cantanhede, e primeyro Marquez de Marialva [UCBG Ms. 593], p. 1-158

PEGADO, César - A perda de Évora em 1663, p. 159-191

FIGUEIREDO, Carlos Proença de - Comentário político da Restauração, p. 193-209

BEAU, Albin Eduard - A ideologia imperialista do Padre António Vieira, p. $211-224$

CASTRO, Luís de - Desagravo de um defensor do Reino (Um inédito de Fr. Francisco de Santo Agostinho Macedo), p. 225-272

IRIA JÚNIOR, Joaquim Alberto - Cartas do Governador e Capitão-mor do Algarve Henrique Correia da Silva, p. 273-300

THOMAS, Henry - Um desconhecido espécime tipográfico do impressor da primeira edição dos «Lusíadas», p. 301-316

MOVIMENTO da Biblioteca Geral da Universidade de Coimbra: 1939, p. [317-319]

MAPA ESTATÍSTICO da Biblioteca Geral da Universidade: ano de 1940, p. [320] MAPA ESTATÍSTICO da Biblioteca Geral da Universidade: ano de 1941, [321] ÍNDICE ao vol. XV, p. [322]

ÍNDICE dos Autores, p. [322]

\section{Vol. XVI (1944)}

PERES, Damião - Palavras de abertura, p. I-II

BRANDÃO, Mário - Alguns documentos relativos a 1580, p. 1-82

RODRIGUES, A. Gonçalves - As cartas da Freira: estudo bibliográfico, p. 83-172

VILHENA, João Jardim de - «A Martinhada», p. 173-179 
CARVALHO, Joaquim de - O livro «Contra os juízos dos astrólogos», p. $181-290$

PIMENTA, Belisário - O «Memorial» de Matias de Albuquerque, p. 291-324

NEVES, Álvaro - Raridades biblíacas: edições incompletas, p. 325-368

PEGADO, César - Adenda à «Martinhada», p. 369

PERES, Damião - Relatórios, p. 371-391

MAPA ESTATÍSTICO da Biblioteca Geral da Universidade: ano de 1942, p. [395] MAPA ESTATÍSTICO da Biblioteca Geral da Universidade: ano de 1943, [396] ÍNDICE ao vol. XVI, p. [397]

ÍNDICE dos Autores, p. [322]

\section{Vol. XVII (1947)}

NEVES, Álvaro - Raridades biblíacas: edições incompletas (continuação do vol. 16), p. 1-82

VILHENA, João Jardim de - Coimbra vista e apreciada pelos estrangeiros, p. 83-266

CRUZ, Maria Lígia - Índices [... dos autores e das espécies que figuram no artigo de João Jardim de Vilhena «Coimbra vista e apreciada pelos estrangeiros»], p. 267-293

SANTOS, Maria Amélia Machado - Bento José, professor de filosofia em Évora, p. 295-300

BANDEIRA, José Ramos - Universidade de Coimbra, p. 301-607

ALMEIDA, Manuel Lopes de - A propósito de «Bento José», professor de filosofia em Évora, p. 609-623

VILHENA, João Jardim de - Uma colecção de ex-libris, p. 625-631

RELATÓRIOS, p. 633-645

MAPA ESTATÍSTICO da Biblioteca Geral da Universidade de Coimbra: ano de 1944, p. [649]

MAPA ESTATÍSTICO da Biblioteca Geral da Universidade de Coimbra: ano de 1945, [650]

MAPA ESTATÍSTICO da Biblioteca Geral da Universidade de Coimbra: ano de 1946, p. [651]

ÍNDICE ao vol. XVII, p. [655]

ÍNDICE dos Autores, p. [655] 


\section{Vol. XVIII (1948)}

BRASÃO, Eduardo - A missão a Roma do Bispo de Lamego, p. 1-65

RAU, Virgínia - Inventário dos bens da Rainha da Grã-Bretanha D. Catarina de Bragança, p. 67-162

LEITE, Serafim - "Colecção dos crimes, e decretos Pelos quaes vinte e hum Jesuitas forão mandados sahir do Estado de Gram Para, e Maranhão antes do exterminio geral de toda a Companhia de Jesus daquelle Estado com Declaração dos mesmos crimes, e resposta a elles, por Manuel Lopes de Almeida», p. 163-293

FERREIRA, Carlos Alberto - lluminuras, aguarelas, ornatos e desenhos à pena dos manuscritos da Biblioteca da Ajuda, p. 294-352

RELATÓRIO, p. 353-359

MAPA ESTATÍSTICO da Biblioteca Geral da Universidade de Coimbra: ano de 1947, p. [363]

ÍNDICE ao vol. XVIII, p. [365]

ÍNDICE dos Autores, p. [365]

\section{Vol. XIX (1950)}

PIMENTA, Belisário - A Campanha de 1801 (Ligeiras considerações a propósito duns documentos), p. 1-44

LUZ, Francisco Mendes da - Relação das rendas da coroa de Portugal feita em 1593 por Francisco Carneiro provedor de ementas da Casa dos Contos, p. 45-108

BRITO, A. da Rocha - O vexame, p. 109-130

ESTEVENS, Manuel Santos - Sinopse cronológica da legislação portuguesa sobre Bibliotecas e Arquivos (1796-1948), p. 131-240

SANTOS, Mariana Amélia Machado - Manuscritos de filosofia do século XVI existentes em Lisboa, p. 241-382

ALMEIDA, M. Lopes de - Um documento sobre a expedição de Du Clerc ao Rio de Janeiro em 1710, p. 383-391

MAPA ESTATÍSTICO da Biblioteca Geral da Universidade de Coimbra: ano de 1948, p. [394]

MAPA ESTATÍSTICO da Biblioteca Geral da Universidade de Coimbra: ano de 1949, p. [396] 
ÍNDICE ao vol. XIX, p. [398]

ÍNDICE dos Autores, p. [398]

\section{Vol. XX (1951)}

CARVALHO, Joaquim de - João Locke, «Ensaio filosófico sobre o entendimento humano», p. 1-212

RODRIGUES, A. Gonçalves - A novelística estrangeira em versão portuguesa no período pré-romântico, p. 213-294

SANTOS, Mariana Amélia Machado - Manuscritos de filosofia do século XVI existentes em Lisboa, p. 295-526

MAPA ESTATÍSTICO da Biblioteca Geral da Universidade de Coimbra: ano de 1950, p. [529]

MAPA ESTATÍSTICO da Biblioteca Geral da Universidade de Coimbra: ano de 1951, p. [531]

ÍNDICE do Volume XX, p. [533]

ÍNDICE de Autores, p. [533]

\section{Vol. XXI (1953)}

LUZ, Francisco Mendes da - Livro das cidades e fortalezas da Índia, p. 1-144

CARVALHO, J. Branquinho de - As festas da canonização da Rainha Santa Isabel promovidas pela Câmara de Coimbra, p. 145-157

PEIXOTO, Jorge - Um novo incunábulo na Biblioteca Geral da Universidade de Coimbra, p. 158-163

DIAS, Luís Fernando de Carvalho - Fr. Heitor Pinto (Novas achegas para a sua biografia), p. 164-344

SERRÃO, Joaquim Veríssimo - Manuel Álvares (1545-1612): um desconhecido português, professor de Medicina na Universidade de Toulouse, p. 345-393

VILHENA, João Jardim de - Coimbra vista e apreciada pelos estrangeiros, p. 394-448

ÍNDICE do Volume XXI, p. [450]

ÍNDICE dos Autores, p. [450] 
MAPA ESTATÍSTICO da Biblioteca Geral da Universidade de Coimbra: ano de 1952 , p. [451]

\section{Vol. XXI (1953) - Suplemento}

LOUREIRO, José Pinto - Índice ideográfico de «O Conimbricense», p. 1-199

\section{Vol. XXII (1955)}

VILHENA, João Jardim de - Coimbra vista e apreciada pelos estrangeiros, p. 1-134

CALADO, Adelino de Almeida - «Dissertação crítica sobre os Estatutos da Universidade de Coimbra [1778]», p. 135-220

SÁ, Artur Moreira de - Pedro Hispano e a crise de 1277 da Universidade de Paris, p. 221-241

SERRÃO, Joaquim Veríssimo - António de Gouveia, professor de Direito em Grenoble, p. 242-341

ALMEIDA, Luís Ferrand de - Informação de Francisco Ribeiro sobre a Colónia do Sacramento, p. 342-445

COSTA, Mário Alberto Nunes - os arquivos del-rei D. António e de seus servidores, p. 446-538

MAPA ESTATÍSTICO da Biblioteca Geral da Universidade de Coimbra: ano de 1953, p. [539]

MAPA ESTATÍSTICO da Biblioteca Geral da Universidade de Coimbra: ano de 1954, p. [540]

ÍNDICE do Volume XXII, p. [541]

ÍNDICE de Autores, p. [541]

ANEXO:

[SALAZAR, António de Oliveira] - Goa e a União Indiana: [discursos], p. 1- 78

\section{Vol. XXIII (1958)}

BOURDON, L. - Jeronimo Osorio et Stanislas Hosius (1565-1578), p. 1-105

BRANDÃO, Mário - Antero de Quental estudante, p. 106-416

RÉVAH, I. S. - La «Descripçam e debuxo do moesteyro de Santa Cruz de

Coimbra», p. 417-420 
ANTÓNIO BRÁSIO (padre) - Novos documentos para a história da Rainha Santa Isabel, p. 421-452

PIMENTA, Belisário - As cartas do Infante D. Pedro à Câmara de Coimbra (1429-1448), p. 453-532

MAPA ESTATÍSTICO da Biblioteca Geral da Universidade de Coimbra: ano de 1955, p. [433]

MAPA ESTATÍSTICO da Biblioteca Geral da Universidade de Coimbra: ano de 1956, p. [434]

ÍNDICE do Volume XXIII, p. [435]

ÍNDICE de Autores, p. [435]

\section{Vol. XXIII (1958) - Suplemento}

«Diccionario bibliographico portuguez : estudos de Innocêncio Francisco da Silva aplicáveis a Portugal e ao Brasil»: Guia bibliográfica por Ernesto Soares, p. 1-762

\section{Vol. XXIV (1960)}

CALADO, Adelino de Almeida - «Livro que trata das cousas da Índia e do Japão», p. 1-138

SERRÃO, Joaquim Veríssimo - Documentos inéditos para a história do reinado de D. Sebastião, p. 139-272

PIMENTA, Belisário - Portalegre e a Guerra da Sucessão, p. 273-301

RIBEIRO, Mário de Sampaio - El-Rei D. João III e o Claustro da Manga do Mosteiro de Santa Cruz de Coimbra, p. 302-337

BRÁSIO, António - Uma carta inédita de Valentim Fernandes, p. 338-358

MAPA ESTATÍSTICO da Biblioteca Geral da Universidade de Coimbra: ano de 1957, p. [359]

MAPA ESTATÍSTICO da Biblioteca Geral da Universidade de Coimbra: ano de 1958, p. [360]

MAPA ESTATÍSTICO da Biblioteca Geral da Universidade de Coimbra: ano de 1959, p. [361]

ÍNDICE do Volume XXIV, p. [363]

ÍNDICE dos Autores, p. [363] 


\section{Vol. XXV (1962)}

GONÇALVES, António Manuel - Historiografia da arte em Portugal, p. 1-64 SERRÃO, Joaquim Veríssimo - Uma Relação do reino de Portugal em 1684, p. $65-170$

RICARD, Robert - Références portugaises chez l'écrivain espagnol Jovellanos (1744- 1811), p. 171-176

VILHENA, João Jardim de - Uma página das minhas Memórias: Amélia Janny, p. 177-187

COSTA, Mário Alberto Nunes - Estremoz e o seu concelho nas «Memórias Paroquiais de 1758», p. 188-351

BRITO, A. da Rocha - Velhas páginas universitárias. I. Galos e Galinhas em doutoramentos universitários de outras eras. II. A vida de um escolar em Medicina no século XVI: Lourenço Vieira, p. 352-394

NEVES, Álvaro - Pseudónimos. Achegas para um Dicionário de Pseudónimos de escritores editados em Portugal, p. 395-409

NOTA BIBLIOGRÁFICA ao M.I. 254, p. 410-411

MAPA ESTATÍSTICO da Biblioteca Geral da Universidade de Coimbra: ano de 1960, p. [413]

MAPA ESTATÍSTICO da Biblioteca Geral da Universidade de Coimbra: ano de 1961, p. [414]

ÍNDICE do Volume XXV, p. [415]

ÍNDICE dos Autores, p. [415]

\section{Vol. XXVI (1964)}

MARQUES, Maria Adelaide Salvador - A Real Mesa Censória e a Cultura Nacional: aspectos da Geografia Cultural Portuguesa no século XVIII, p. 1-207

CARVALHO, José G. Herculano de - Um Tipo Literário e Humano do Barroco, p. 208-227

GAMA, Eurico - Catálogo dos Pergaminhos do Arquivo Municipal de Elvas, p. 228-361

RIBEIRO, Mário de Sampaio - A «Arte de Cantollano», de autor desconhecido (R. 14 670), da Biblioteca Nacional de Madrid e a «Arte» de Juan Martinez, p. 362- [396] 
ÍNDICE do Volume XXVI, p. [497]

ÍNDICE dos Autores, p. [497]

\section{Vol. XXVII (1966)}

CALADO, Adelino de Almeida - Frei João Álvares: estudo textual e literário-cultural, p. 1-387

ALMEIDA, Manuel Lopes de - Subsídios para a história da Universidade de Coimbra, p. 389-539

OLIVEIRA, António de - A livraria de um teólogo do século XVI, p. 541-588 ÍNDICE do Volume XXVII, p. [589]

ÍNDICE dos Autores, p. [589]

\section{Vol. XXVIII (1970)}

GAMA, Eurico - Cartas de Leite de Vasconcellos ao bibliófilo António José Torres de Carvalho, p. 1-38

OLIVEIRA, António de - A livraria de um canonista do século XVII, p. 39-82 LIMA, Jorge Hugo Pires de - Processos políticos do reinado de D. Miguel, p. 83-651

ÍNDICE do Volume XXVIII, p. [653]

ÍNDICE dos Autores, p. [653]

\section{Vol. XXIX (1972)}

MANUPPELLA, GIACINTO - Bibliografia degli scritti di Max Leopold Wagner, p. 1-141

ALMEIDA, Manuel Lopes de - Artes e ofícios em documentos da Universidade, p. 143-348

VIQUEIRA, José M. - Camões y su hispanismo, p. 349-445

CUNHA, Rosalina da Silva - Subsídios para a história da conservação do peixe em Portugal, p. 446-514

ÍNDICE do Volume XIX, p. [515]

ÍNDICE dos Autores, p. [515] 


\section{Vol. XXX (1973)}

MADEIRA, Eugénio Tomás Mendes - Obras impressas em Portugal nos séculos XVI e XVII, p. 1-96

SOUSA, José Manuel Motta de - O Catálogo Colectivo da Universidade de

Coimbra 1967-1972, p. 97-116

RELAÇÃO DA ENTRADA que fez o bispo do Rio de Janeiro D. Fr. António do

Desterro Malheiro nesta cidade em 1747, p. 117-141

EXPOSIÇÃO Camoniana, p. 142-320

CRUZ, Guilherme Braga da - Homenagem ao Diretor-Geral cessante do

Ensino Superior e das Belas-Artes Dr. João de Almeida, p. 321-330

PEGADO, César - Relatório respeitante ao ano de 1970, p. 331-375

CRUZ, Guilherme Braga da - Relatório respeitante ao ano de 1971, p. 377-440

\section{Vol. XXXI (1974)}

AZEVEDO, Maria Antonieta Soares de - O Prior do Crato, Filipe Il de Espanha e o trono de Portugal: algumas notas bibliográficas (século XVI), p. 1-54 MENDES, Maria Teresa Pinto - Fundos especiais da Biblioteca Geral da Universidade, p. 55-88

PEIXOTO, Jorge - ISBD (M e S): a descrição bibliográfica internacional normalizada das monografias e das publicações em série, p. 89-188

LEMOS, Maria Luísa - Secção de Manuscritos da Biblioteca Geral da Universidade de Coimbra: Manuscritos 3161-3230, p. 189-256

PERICÃO, Maria da Graça ; FARIA, Maria Isabel Ribeiro de - Edições quinhentistas de Damião de Góis e de André de Resende existentes na Biblioteca Geral da Universidade de Coimbra, p. 257-328 BIBLIOGRAFIA de Belisário Pimenta, p. 329-495

\section{Vol. XXXII (1975)}

MIRANDA, José da Costa - Uma tradução portuguesa, manuscrita, inédita (século XIX) de «Esther», de Racine?, p. 1-16

CRUZ, Maria Helena Braga da, PERICÃO, Maria do Rosário, CASTRO, Zília Maria Osório - Publicações periódicas portuguesas: respigo de artigos, 17-58 
BARBOSA, José - Molière, p. 59-144

FARIA, Maria Isabel Ribeiro de ; PERICÃO, Maria da Graça - Índice do «Mercúrio Português», p. 145-272

MADEIRA, Eugénio Tomás Mendes - Obras impressas em Portugal nos séculos XVI e XVII, p. 273-385

\section{Vol. XXXIII (1977)}

MIRANDA, José da Costa - Teatro italiano, manuscrito (século XVIII): sobre alguns textos existentes em Bibliotecas e Arquivos Portugueses, p. 1-24 FARIA, Francisco - Quatro responsórios de Natal de D. Pedro da Esperança, p. 25-68

SÁ, A. Moreira de - Livros de uso de Frei Diogo de Murça, p. 69-110

CRUZ, Lígia - O legado da biblioteca de João Pedro Ribeiro à Universidade, p. 111-154

DIAS, Luís Fernando de Carvalho - Inéditos de António Ribeiro dos Santos, p. $155-234$

PERICÃO, Maria da Graça ; FARIA, Maria Isabel Ribeiro de - Inquisição, p. 235-441

\section{Vol. XXXIV (1978) - 1a parte}

FERNANDES, Raul de Matos - Jornais do Porto (1896-1925), p. 1-112 HUMPHREY, K. W. - The crisis for Academic Libraries in Southern Europe, p. 113-126

MIRANDA, José da Costa - Luigi Pulci, Durante da Gualdo e Cieco da Ferrara: apontamentos sobre a presença, em Portugal, dos seus poemas cavaleirescos, p. 127-144

GODINHO, António Matos - Medlars e Medline, p. 145-182

SOUSA, Maria Armanda de Almeida e - Ser bibliotecário hoje, p. 183-188 GONÇALVES, Maria da Conceição Osório - Alguns aspectos dos custos da automatização em bibliotecas e serviços de informação, p. 189-194 MOREIRA, Alzira Teixeira Leite - O instrumento de busca ao serviço do investigador, p. 195-202

CARMO, M. de Lurdes Akola da Cunha Meira do - O índice alfabético de assuntos do Catálogo C.D.U. da Biblioteca Nacional de Lisboa, p. 203-212 
PEREIRA, Isaías da Rosa - Livros de aniversários de Santa Maria da Alcáçova de Santarém e de Santiago de Coimbra, p. 213-240

SUMÁRIOS das Publicações Periódicas Portuguesas: projecto de publicação, p. 241-270

FARIA, Maria Isabel Ribeiro de - Diderot «Carta histórica e política sobre o comércio do livro», p. 271-354

COELHO, Urbano Domingues - Regras de colocação de monografias, p. 355-376

PEREIRA, Marcelino Rodrigues - Alguns conceitos básicos de Arquivística moderna, p. 377-407

\section{Vol. XXXIV (1978) - 2a parte}

ALBUQUERQUE, Luís de - Um exemplo de «Cartas de serviços» da Índia, p. 1-12

RAMALHO, Américo da Costa - Alguns aspectos da introdução do humanismo em Portugal, p. 13-34

CASTRO, Aníbal Pinto de - António Nobre, Alberto de Oliveira e o editor França Amad : correspondência inédita, p. 35-56

BRITO, Maria Fernanda de - Pedro Nunes na tipografia de quinhentos, p. 57-74

BELLOTTO, Heloísa Liberalli - O Morgado de Mateus, Governador de São Paulo, p. 75-140

FARIA, Francisco Leite - Livros impressos em Portugal no século XVI existentes na Biblioteca Nacional do Rio de Janeiro, p. 141-226

$\mathrm{HORCH}$, Rosemarie Erika - Livros quinhentistas portugueses existentes no Instituto de Estudos Brasileiros da Universidade de São Paulo, Brasil, p. 227-242

MENDES, Maria Teresa Pinto - O Bibliotecário e a sua circunstância, p. 243258

ACHEGAS para umas regras portuguesas de alfabetação, p. 259-298 IFLA ; RAPOSO, Maria Emília - ISBD(M), p. 299-392

IFLA ; COSME, Carlos Dinis (trad.) ; MARTINS, Paula Maria Fernandes (introd.) - ISBD(S), p. 393-536 


\section{Vol. XXXIV (1979) - 3. ${ }^{\text {a }}$ parte}

ANDRADE, Maria Francisca de Oliveira ; ANDRADE, António Alberto Banha de - Subsídios para a história da aula de árabe no Convento dos Terceiros de S. Francisco, p. 1-22

PERICÃO, Maria da Graça - A Livraria Visconde da Trindade, p. 49-70

CUNHA, Maria de Fátima Vila Pouca e - Subsídios para a bibliografia de autores portugueses referente a Pedro Nunes, p. 71-106

DIAS, Manuel Nunes - O sistema das capitanias do Brasil, p. 107-134

MARIANO, Emília Henriques Gouveia da Silva - Pequena achega para conservação de documentos gráficos, p. 135-140

PUGLIESI, Haidée Marquiafave - $\mathrm{A}$ «avería» nos livros de contas do Arquivo Municipal da Venezuela, p. 141-152

FARIA, Francisco - Algumas notas sobre os manuscritos musicais da Biblioteca

Geral da Universidade de Coimbra, p. 153-164

MELO, Ana Maria Osório Pereira de - Apostilas, p. 165-218

VAZ, Maria Laurinda dos Reis Antunes - Catálogo da colecção de medalhas da Biblioteca Geral da Universidade de Coimbra, p. 249-342

CARRATO, José Ferreira - O Marquês de Pombal e a reforma dos estudos menores em Portugal, p. 343-384

CATARINO, Maria de Lurdes - Os ex-libris portugueses da colecção João Jardim de Vilhena da Biblioteca Geral da Universidade de Coimbra, p. 385-432

OLIVEIRA, Maria Manuela Rodrigues Nobre - A colecção de mapas da Biblioteca Geral da Universidade de Coimbra, p. 433-436

LEMOS, Maria Luísa - Impressos musicais da Biblioteca Geral da Universidade de Coimbra, p. 437-575

\section{Vol. XXXV (1980)}

MOTA, A. Teixeira da - Cartas antigas da Índia existentes em Portugal (séculos XVIII, XIX e XX), p. 1-122

MIRANDA, José da Costa - Ludovico Ariosto, «Commedie»: algumas considerações e alguns apontamentos acerca da sua presença em Portugal, p. $123-138$ 
CAEIRO, Francisco da Gama - Livros e livreiros franceses em Lisboa, nos fins de setecentos e no primeiro quartel do século XIX, p. 139-168 ANDRADE, Isabel Maria Freire de - Ensaio de catalogação de ex-libris, p. $169-282$

MALDONADO, Maria Hermínia - Uma fábula burlesca de D. Francisco de Portugal, p. 283-322

ANDRADE, Carlos Santarém - «Presença»: uma revista, um movimento, p. 323-394

\section{Vol. XXXVI (1981)}

COSTA, Avelino de Jesus da - Inventário dos bens e obituário de Santa Maria da Alcáçova de Santarém, p. 1-30

MELO, Ana Maria Osório Pereira de ; SOUSA, Maria Armanda de Almeida e - Bibliografia ex-librística anexa à colecção J. J. Vilhena da Biblioteca Geral da Universidade de Coimbra, p. 31-46

MIRANDA, José da Costa - Torquato Tasso, «Gerusalemme liberata»: a intervenção da censura inquisitorial portuguesa, p. 47-56

LIMA, Ebion de - Os oratorianos e a polémica da Gramática Latina no século XVIII, p. 57-72

MOREIRA, Alzira Teixeira Leite - Publicações impressas nos séculos XVI, XVII e XVIII existentes na Biblioteca do Tribunal de Contas, p. 73-112

SOLLA, Luiz de Castro e - A Revolta Liberal de 1828 (novos aspectos), p. 113-136

REGRAS de alfabetação, p. 137-201

CATÁLOGO DOS RESERVADOS da BGUC. Suplemento, p. 203-252

MADEIRA, E. T. Mendes - Obras impressas em Portugal nos séculos XVI e XVII, p. 253-324

ALBUQUERQUE, Luís de - Relatório de actividades, p. 325-342

ALBUQUERQUE, Luís de - Homenagem ao Doutor Manuel Lopes de Almeida, p. 343-346

ALBUQUERQUE, Luís de ;CASTRO, Aníbal Pinto de - Homenagem ao Doutor Braga da Cruz, p. 347-366 


\section{Vol. XXXVII (1982)}

SOLLA, Luiz de Castro e - O ouro do Brasil nos arquivos do século XVIII, p. $5-56$

CARITA, Rui - A Planta do Funchal de Mateus Fernandes (c. 1570), p. 57-108 SANTOS, Maria Emília Madeira - Silva Porto e os problemas da África Portuguesa no século XIX, p. 109-132

BARRETO, Manuel Saraiva - Uma «Ars eloquentiae» dos primórdios do humanismo em Portugal, p. 133-160

OLIVEIRA, Eduardo Pires de - Estudos bracarenses: para uma bibliografia do Cónego Arlindo Ribeiro da Cunha, p. 161-188

MIRANDA, José da Costa - Ecos de Torquato Tasso, «Gerusalemme liberata», na Academia dos Generosos, de Lisboa: achegas para um (lendário) conflito literário seiscentista, p. 189-200

KHOURY, Ibrahim (trad. e notas) - As-Sufaliyya «The poem of Sofala», p. 201-332

PEIXEIRO, Horácio Augusto ; ALVES, Luísa Maria Picciochi Azevedo - Conclusões do «Levantamento das condições gerais de conservação dos documentos gráficos do nosso património bibliográfico e documental - Apreciação estatística», p. 333-352

\section{Vol. XXVIII (1983)}

COSTA, Avelino de Jesus da - A Biblioteca e o Tesouro da Sé de Coimbra nos séculos XI a XVI, p. 1-220

FERNANDES, Rogério - Luís da Silva Mousinho de Albuquerque e as Reformas do Ensino em 1835-1936, p. 221-304

LIMA, Ebion de - Notícia biobibliográfica sobre a família Montebelo, $p$. 305-318

PAYSAC, Henry de - Eugène de Castro et Francis Vielé-Griffin: une amitié symboliste, p. 319-336

\section{Vol. XXXIX (1984)}

SANTOS, Maria Emília Madeira - O estudo da hidrografia numa região de civilizações da terra, a África Austral, p. 1-18 
ALMEIDA, A. A. Marques de - Herança e inovação no capitalismo em Portugal (século XIV-XVIII): a escrituração por partidas dobradas, p. 19-40 Correspondência de Luciano Pereira da Silva para Joaquim de Carvalho, p. $41-90$

GUERREIRO, Inácio - A Sociedade Real Marítima e o exame das cartas hidrográficas. Censura da Carta de Cabo Verde, de Francisco António Cabral (1790), p. 91-142

RANDLES, W. G. L. - Portuguese and Spanish attempts to measure longitude in the 16th century, p. 143-160

OLIVEIRA, Eduardo Pires de - O contributo do jornal «Cerveira nova» para o estudo e divulgação do património cultural cerveirense, p. 161-168

NAGEL, Rolf - Em tempo de mudança: um conceito activo de arquivo nacional, p. 169-176

BREMNER, R. W - The construction and origins of two Portulan charts, p. 177-192

IFLA ; RAPOSO, Maria Emília (trad.) ; MARTINS, Paula Fernandes (nota intr.) - ISBD (G), p. 193-260

\section{Vol. XL (1985)}

LEMOS, Maria Luísa - A literatura autonomista no século XVII, através do Códice 29 da Biblioteca Geral da Universidade, p. 3-94

CÓDICE 29. Primeira parte. Do grande e aparatoso recebimento que a ... cidade de Évora fez ao duque de Bragança, p. 95-206

CÓDICE 29. Segunda parte. Composição dramática. Das tribulações que afligiram o Reino de Portugal no ano de 1637, p. 207-359

Vol. XLI (1992)

COSTA, Mário Alberto Nunes - Em torno da correspondência de Diogo Barbosa Machado, p. 1-104

LEMOS, Maria Luísa - A literatura autonomista no século XVII: glossário dos textos, p. 105-190

CARVALHO, Rui Galvão de - O espólio poético do Doutor Vicente José Ferreira Cardoso da Costa, p. 191-208

MARQUES, J. J. Dias - Duas notas à margem do «Amor de perdição», p. 209-226 
MATOS, Manuel Cadafaz de - Leopoldo Battistini, um pré-rafaelita italiano na dimensão do seu classicismo, p. 227-248

MENDES, Dulce Geraldes - Uma carta de Antero de Quental, p. 249-264 MARIANO, Emília Gouveia (transcr.) - Pharmacopea geral. Segunda parte:

Dos medicamentos preparados e compostos, p. 265-348

IFLA ; GONÇALVES, Maria da Conceição (trad.), RAPOSO, Maria Emília (trad.)

- Princípios para a aplicação das ISBDs à descrição das partes componentes, p. 349-406

MALDONADO, Maria Hermínia - António da Fonseca Soares (Frei António das Chagas). Trinta romances inéditos, p. 407-496

SOUSA, José Manuel Motta - As publicações em série: passado, presente e perspectivas futuras. Algumas reflexões a propósito da problemática existente, p. 497-514

CASTRO, Aníbal Pinto de - Relatório ano lectivo 1990-1991, p. 515-534

CASTRO, Aníbal Pinto de - VII Centenário da Universidade de Coimbra: exposição bibliográfica... Discurso do Director da Biblioteca, p. 535-540

\section{Vol. XLII (1994)}

CALADO, Adelino Almeida - «Livro de Exopo». Edição crítica com introdução e notas, p. 1-100

COSTA, Mário Alberto Nunes - A «Breve recopilação... da fundação, antiguidades e excelências... de Estremós», p. 101-164

LOURENÇO, Carlos Rogenmoser - Apontamentos sobre a navegação e a cartografia no dealbar dos descobrimentos marítimos, p. 165-188

RAMOS, Fernando - A intersubjectividade em Gabriel Marcel, p. 189-212

SOUSA, Maria Armanda de Almeida e - Campos de Figueiredo, 1895-1965: biografia literária e bibliografia de um valor esquecido, p. 213-252 GOMES, Saul António - Corregedores da Comarca da Estremadura e suas intervenções no Concelho de Leiria na Idade Média, p. 253-280

REIS, A. Estácio dos - Old globes in Portugal, p. 281-298

GOUVEIA, Valdemar Fernando Peça de Araújo - Os livros da Imprensa da Universidade de Coimbra depositados na Biblioteca da Universidade, p. 299-306

VELOSO, Lúcia - O projecto AIDA no contexto de um serviço nacional de empréstimo interbibliotecas, p. 307-318 
PERICÃO, Maria do Rosário - Informatização e cooperação numa rede de

bibliotecas: a propósito do Projecto do SIIB/Centro, p. 319-334

CASTRO, Aníbal Pinto de - Relatório ano lectivo de 1991-1992, p. 335-458

CASTRO, Aníbal Pinto de - Relatório ano lectivo de 1992-1993, 359-381

CASTRO, Aníbal Pinto de - Sessão comemorativa do $1{ }^{\circ}$ Centenário do nascimento do Doutor Joaquim de Carvalho... Discurso do Director da Biblioteca, p. 383-398

COXITO, Amándio - Joaquim de Carvalho: antipositivismo e metafísica, p. 399-409

CASTRO, Aníbal Pinto de - Inauguração da Sala Belisário Pimenta. Discurso do Director da Biblioteca, p. 411-416

CASTRO, Aníbal Pinto de - Exposição bibliográfica consagrada ao Doutor Manuel de Paiva Boléo ... Discurso do Director da Biblioteca, p. 417-421

CASTRO, Aníbal Pinto de - Sessão inaugural da XII Reunião do Grupo de Utilizadores DOBIS/LIBIS. Discurso do Director da Biblioteca, p. 423-428

\section{Vol. XLIII (1997)}

PINTO, Eduardo Vera-Cruz - Para a grafia jurídica medieval: Cambiglioni versus Cambiglioni, p. 1-20

COSTA, Mário Alberto Nunes - Heurística e historiografia na Academia Real da História Portuguesa, p. 21-66

COSTA, Jaime Raposo - O liberalismo vintista e o Brasil (1820-1822), p. 67-86 MATOS, Manuel Cadafaz de - D. Frei Alexandre de Gouveia: da Universidade de Coimbra ao bispado de Pequim, p. 87-112

SOARES, Teresa Luso; SOARES, Miguel Luso; SOARES, Fernando Luso - «As antiguidades da Lusitânia» de André de Resende: notas de leitura a propósito da Introdução..., p. 113-122

ALMEIDA, Aníbal - A «Universidade de Coimbra», o «culto do diabo» e uma noção de «caranguejo», p. 123-128

IFLA ; RAPOSO, Maria Emília (trad.) ; GONÇALVES, Maria da Conceição Osório (trad.) - ISBD(M), p. 129-272

MACHADO, Ana Mafalda de Sousa - A conversão retrospectiva na Biblioteca Geral da Universidade de Coimbra: breves considerações, p. 273-280 
FRANÇA, Isabel; DIAS, Leonor Marinho; VELOSO, Lúcia; PERICÃO, Maria do Rosário; CANAS, Noémia; MARTINS, Paula Fernandes - Linhas gerais para uma política de informação na Universidade de Coimbra, p. 281-294 VIDA DA BIBLIOTECA, p. 295-335

\section{Vol. XLIV (2010) - Formato eletrónico}

FIOLHAIS, Carlos - Palavras de apresentação

O SERVIÇO PÚBLICO da Biblioteca da Universidade: roteiro da Mostra

ESTUDANTE, Paulo - Para um maior protagonismo da Universidade de Coimbra perante o seu património musical

ROSETE, Marta Lopes - Estudo da integração de espólios na Biblioteca

Geral da Universidade de Coimbra: 1985-1995

AMARAL, A. E. Maia do ; GOMES, Virgínia - Obra de José Contente (19071957) no património da Biblioteca Geral

PEREIRA, Maria José Otão da Silva - Liber chronicarum ou Crónica de Nuremberga (1493)

LIMA, Ebion de - Uma fruta para o Grão-Duque: carta do Padre António Vieira a Cosme III

MORAIS, Carlos Eduardo Mendes de - Guia de fontes primárias sobre académicos esquecidos e renascidos 1724-1759

NOVO REGULAMENTO da BGUC e do SIBUC

CARTA AOS MEMBROS do CG da UC pelo Diretor da BGUC e do SIBUC...

OUTROS TESOUROS da Biblioteca Geral: roteiro da Mostra apresentada ao CG da UC

RELATÓRIO de atividades: 2008-2009

\section{Vol. XLV (2014) - Formato eletrónico}

BERNARDES, José Augusto Cardoso - Uma história para a História das bibliotecas da Universidade

FERREIRA, Carla - A automatização da(s) Biblioteca(s) da Universidade de Coimbra 